\title{
Adolescents' Social Media Experiences and Their Self-Esteem: A Person-Specific Susceptibility Perspective
}

\author{
Patti M. Valkenburg ${ }^{1}$, J. Loes Pouwels ${ }^{1}$, Ine Beyens ${ }^{1}$, Irene I. van Driel ${ }^{1}$, and Loes Keijsers ${ }^{2}$ \\ ${ }^{1}$ Amsterdam School of Communication Research, University of Amsterdam \\ ${ }^{2}$ Department of Psychology, Education, and Child Studies, Erasmus University
}
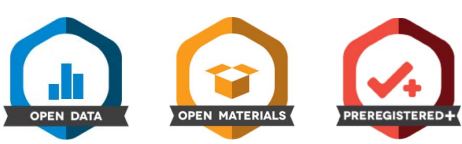

The aim of this preregistered study was to compare and explain the effects of (a) time spent on social media (SM) and (b) the valence (positivity or negativity) of SM experiences on adolescents' self-esteem. We conducted a 3-week experience sampling (ESM) study among 300 adolescents (13-16 years; 126 assessments per adolescent; 21,970 assessments in total). Using an $N=1$ method of analysis (Dynamic Structural Equation Modeling [DSEM]), we found that the within-person effects of time spent with SM on selfesteem ranged from strongly negative $(\beta=-.31)$ to moderately positive $(\beta=+.27)$ across adolescents. Across all ESM observations of the valence of adolescents' SM experiences, $55 \%$ of these experiences were positive, $18 \%$ negative, and $27 \%$ neutral. Finally, 78\% of adolescents experienced a positive within-person effect of the valence of SM experiences on self-esteem $(\beta \geq+.05), 19 \%$ no to a very small effect $(-.05<\beta<+.05)$, and $3 \%$ a negative effect $(\beta \leq-.05)$. These sizeable differences in person-specific effects could be explained by adolescents' self-esteem level, self-esteem instability, and their tendency to base their self-esteem on peer approval.

Keywords: intensive longitudinal data, differential susceptibility, Instagram, Snapchat, Dynamic Structural Equation Modeling (DSEM)

Supplemental materials: https://doi.org/10.1037/tmb0000037.supp

Acquiring self-esteem, the positive and relatively stable evaluation of the self, is a central developmental task in adolescence. Selfesteem may induce adolescents to try out new things, be open to learning and feedback, take calculated risks, and, by doing so, explore their potential. Self-esteem has been positively linked to a healthy peer attachment (Gorrese \& Ruggieri, 2013), life satisfaction (Proctor et al., 2009), and success later in life (Orth \& Robins, 2014). In the past decade, over a dozen empirical studies have
Action Editor: Nick Bowman was the action editor for this article.

ORCID iDs: Patti M. Valkenburg (iD https://orcid.org/0000-00030477-8429; J. Loes Pouwels (iD https://orcid.org/0000-0002-9586-392X; Ine Beyens (iD https://orcid.org/0000-0001-7023-867X; Irene I. van Driel (iD) https://orcid.org/0000-0002-7810-9677.

This 3-week experience sampling (ESM) study is part of a larger longitudinal study on the psychosocial consequences of social media use among middle adolescents. It uses data from (a) the second 3-week ESM wave, which was fielded in June 2020 and (b) two biweekly surveys around this ESM wave. The study builds upon an earlier ESM study on social media use and self-esteem by Valkenburg, Beyens, et al. (2021a), which used data from the first 3-week ESM wave, fielded in November/ December 2019.

A full overview of all preprints and published papers of the larger project can be found on our project website (https://www.project-awesome.nl/ publications).

Data Availability: The anonymous data set on which this article is based is published on Figshare (Valkenburg, Pouwels, et al., 2021).

Conflicts of Interest: The authors declare that there is no conflict of interest.

This study was funded by an NWO Spinoza Prize and a Gravitation grant (NWO Grant 024.001.003; Consortium on Individual Development) awarded to Patti M. Valkenburg by the Dutch Research Council (NWO).
Additional funding was received from a VIDI grant (NWO VIDI Grant 452.17.011) awarded to Loes Keijsers.

We would like to thank Tim Verbeij and Teun Siebers for their contribution to the data collection of this study.

Open Science Disclosures:

II The data are available at https://doi.org/10.21942/uva.14095971

* The analysis scripts and materials are available at https://osf.io/75k4x/ $\checkmark$ The preregistered design and sampling plan of the larger project is accessible at https://osf.io/327cx

The preregistration of the hypotheses and analysis plan of the current study is available at https://osf.io/43m7t.

Open Access License: This work is licensed under a Creative Commons Attribution-NonCommercial-NoDerivatives 4.0 International License (CC-BYNC-ND). This license permits copying and redistributing the work in any medium or format for noncommercial use provided the original authors and source are credited and a link to the license is included in attribution. No derivative works are permitted under this license.

Disclaimer: Interactive content is included in the online version of this article.

Contact Information: Correspondence concerning this article should be addressed to Patti M. Valkenburg, Amsterdam School of Communication Research, University of Amsterdam, Spui 21, Amsterdam 1012 CX, The Netherlands. Email: p.m.valkenburg@uva.nl 
examined the effects of social media (SM) use on adolescents' selfesteem (e.g., Barthorpe et al., 2020; Cingel \& Olsen, 2018; Meeus et al., 2019; Rodgers et al., 2020; Valkenburg, Beyens, et al., 2021a). In addition, two meta-analyses (Huang, 2017; Liu \& Baumeister, 2016) have tried to integrate the results of these studies, both yielding very small to small negative pooled associations of SM use with self-esteem ( $r=-.04$, ns, Huang, 2017; $r=-.09$, $p<.01$, Liu \& Baumeister, 2016).

A recent experience sampling (ESM) study, based on an earlier ESM wave among the same sample of adolescents as in the present study (see Method section), has attempted to explain these small pooled associations (Valkenburg, Beyens, et al., 2021a). In this ESM study, Valkenburg, Beyens, et al. employed a so-called person-specific, $N=1$ method of analysis (McNeish \& Hamaker, 2020), which allowed them to investigate the unique effects of time spent on SM on each single adolescent's self-esteem (i.e., by computing a unique effect size for each adolescent). Their study confirmed the weak overall effect of time spent on SM on selfesteem reported in the meta-analyses. But it also revealed substantial differences in the person-specific effects: Whereas most adolescents were not or hardly affected by their time spent on SM, a small group experienced positive effects, and another small group experienced negative effects on self-esteem. Indeed, these preliminary findings suggest that the weak pooled effects reported in the meta-analyses may have been small because they involve overall, average effects resulting from heterogeneous samples of "nonsusceptibles," "positive susceptibles," and "negative susceptibles."

While promising and insightful, earlier work on the effects of SM use on self-esteem leaves two important gaps that, if filled, could further improve our understanding of this effect. First, many previous studies, including Valkenburg, Beyens, et al. (2021a), have investigated how time spent on SM could affect adolescents' selfesteem. It is possible, though, that time spent on SM may be too "neutral" to arrive at a true understanding of the effect of SM use on self-esteem. After all, most self-esteem theories emphasize that it is the valence (the positivity or negativity) rather than the duration of experiences that predict fluctuations in self-esteem. It is assumed that self-esteem surges when we succeed or when others accept us and drops when we fail or when others reject us (Leary \& Baumeister, 2000). Therefore, the first aim of the present study was to compare the predictive contribution of (a) adolescents' time spent on SM and (b) the valence (the positivity or negativity) of their SM experiences.

A second gap in the literature is a lack of understanding of the factors that may explain potential differences in the effects of SM use on self-esteem. Earlier studies have predominantly investigated the moderating role of gender in the SM-self-esteem relation (e.g., Blomfield Neira \& Barber, 2014; Meeus et al., 2019), mostly yielding nonsignificant results. In the present study, we extended these earlier studies with four additional factors that may moderate the SM-self-esteem association: Self-esteem level, self-esteem instability, peer approval contingency (i.e., the extent to which adolescents' self-esteem depends on peer approval), and physical appearance contingency (i.e., the extent to which their self-esteem depends on their physical appearance, Crocker \& Wolfe, 2001).

To address the two aims of our study, we employed a preregistered 3-week ESM study among 300 middle adolescents, whom we surveyed six times a day (126 measurement moments per person;
21,970 observations in total). We focused on middle adolescence because this is the period of most significant fluctuations in selfesteem (Harter, 2012). Before the start of our study, we conducted a national survey study among 1,000 middle adolescents (van Driel et al., 2019). This survey identified Instagram, Snapchat, and WhatsApp as the three most popular platforms among this age group. From this survey and a series of pilot interviews, we learned that adolescents typically use these platforms in complementary ways, for example, to present themselves to their broader circle of friends (Instagram, Snapchat), to have fun with their friends (Snapchat), and to exchange more intimate information with close friends or family (WhatsApp). Both these public, asynchronous selfpresentations and these private, synchronous exchanges with close friends have been shown to affect adolescents' self-esteem (Steinsbekk et al., 2021; Valkenburg et al., 2017).

To capture adolescents' person-specific susceptibilities to the effects of time spent on SM and the valence of SM experiences on self-esteem, we employed Dynamic Structural Equation Modeling (DSEM). DSEM combines the strengths of multilevel analysis and Structural Equation Modeling with $N=1$ time-series analysis (Asparouhov et al., 2018). The $N=1$ time-series part of DSEM enabled us to establish the longitudinal, within-person effects of time spent with SM and the valence of SM experiences on the selfesteem of each single adolescent. And it also allowed us to investigate the between-person differences (i.e., the heterogeneity) in these within-person effects.

Within-person effects indicate to what extent SM use leads to changes in a person's self-esteem as a result of this person's SM use. Between-person associations indicate whether persons with high SM use have lower (or higher) self-esteem than persons with low SM use. In other words, within-person analyses compare SMinduced changes in self-esteem in a person with this person's average self-esteem score (i.e., one's "true" score, Nesselroade, 1991, p. 229). Between-person analyses compare the SM-induced self-esteem scores of a person with those of other persons. Withinperson methods of analysis are generally better attuned to investigate (social) media effects than between-person methods of analysis. After all, a media effect is an intraindividual change within a person due to the media use of this person (Valkenburg et al., 2016), and such changes can therefore best be investigated with within-person methods of analysis.

\section{The Valence of Social Media Experiences}

Even though most empirical studies have examined the associations of time spent on SM with self-esteem, most self-esteem theories emphasize that it is the valence of experiences (rather than their duration) that induces ups and downs in self-esteem (Crocker \& Brummelman, 2018; Rosenberg, 1986). For example, Leary and Baumeister's (2000) sociometer theory argues that selfesteem serves as a sociometer that gauges the level of approval or disapproval from one's social environment. Self-esteem goes up when people succeed or when others accept them, and it drops when they fail or when others reject them. Sociometer theory conceptualizes self-esteem as an affectively laden evaluation of the self, meaning that changes in self-esteem are inextricably connected to changes in affect (e.g., feelings of pride and triumph vs. feelings of guilt and embarrassment). 
Self-esteem theories acknowledge sizeable individual differences in how the self-esteem of individuals can be affected. These theories argue that some people may experience significant boosts (or drops) in self-esteem in response to minor positive (or negative) experiences, whereas others may only experience self-esteem fluctuations in case of severe self-relevant experiences (Crocker \& Brummelman, 2018; Harter \& Whitesell, 2003). Such individual differences in susceptibility to experiences is also proposed in the differential susceptibility to media effects model (Valkenburg \& Peter, 2013) and other media effects theories that postulate that media users can differ greatly in their susceptibility to the effects of their media experiences (for a review, see Valkenburg et al., 2016).

The aim of this study was to contribute to media effects and selfesteem theories by investigating and comparing the effects of time spent on SM and the valence of SM experiences on self-esteem, and to explain these effects. Several earlier studies suggest that the positivity or negativity of social media experiences may be a more important predictor of psychosocial outcomes than time spent on social media (e.g., Orben \& Dunbar, 2017; Primack et al., 2019). However, because virtually all earlier studies included time spent on $\mathrm{SM}$ as the predictor of self-esteem (for a review, see Valkenburg, Beyens, et al., 2021a), for reasons of comparability, we also included time spent on SM in the present study.

Based on the weight of evidence in earlier SM-self-esteem studies, we hypothesized that adolescents who spend more time on SM report lower levels of self-esteem than adolescents who do less so (between-person hypothesis, H1). And on the basis of the result of the earlier ESM study by Valkenburg, Beyens, et al. (2021a), we hypothesized significant between-person differences in the within-person effects of time spent with SM on self-esteem (H2). Furthermore, we expected that adolescents whose experiences on SM are more positive report a higher self-esteem level than adolescents whose experiences are less positive (between-person hypothesis, H3). Finally, we expected that, overall, the more positive adolescents' SM experiences were in the previous hour, the stronger their increase in self-esteem would be (within-person hypothesis, H4). Finally, we expected significant between-person heterogeneity in this within-person effect (H5).

\section{Investigating Potential Moderators}

Even though a detection of person-specific effects of time spent on SM and the valence of SM experiences on self-esteem is valuable in its own right, it does not answer the question of why self-esteem goes up for some and goes down for others in response to similar levels of time spent on SM and similarly valenced SM experiences. To answer this question, we investigated the moderating roles of (a) gender, (b) self-esteem level, (c) self-esteem instability, (d) peer approval contingency, and (e) appearance contingency. To do so, we assessed, in a first step, the person-specific effects of time spent on SM and the valence of SM experiences on self-esteem, and in a second step we studied whether and how these five potential moderators predict these person-specific effects. Several methodologists have called for such a moderation approach to avoid ecological fallacies in the interpretation of results, that is, deriving conclusions about individuals based on analyses of group data (e.g., Lerner \& Lerner, 2019). We developed research questions rather than hypotheses to investigate the predictive roles of each of the five moderators because earlier work either did not find any moderating effect on the relationship of adolescents' SM use and self-esteem (in the case of gender) or did not investigate such effects (in the case of the remaining four moderators).

\section{Gender}

Five earlier studies have investigated the moderating role of gender in the between-person relation of SM use and self-esteem. Four of these studies found a nonsignificant effect (Blomfield Neira \& Barber, 2014; Kelly et al., 2019; Košir et al., 2016; Meeus et al., 2019), while one study found a stronger negative SM-self-esteem relation among girls than boys (Barthorpe et al., 2020). It is conceivable that adolescent boys and girls differ in their susceptibility to the effects of time spent on SM and the valence of SM experiences on self-esteem. Adolescent girls generally display somewhat lower levels of selfesteem than adolescent boys (Harter, 2012), report somewhat more frequent social media use (Pew Research Center, 2018), and are somewhat more sensitive to social influences on their self-esteem (Meier et al., 2011). Therefore, we investigated to what extent the within-person effect of time spent with SM on self-esteem (RQ1a) and that of the valence of SM experiences (RQ1b) would depend on adolescents' gender.

\section{Self-Esteem Level and Instability}

The literature offers two opposite hypotheses that consider the effect of social media use on self-esteem: A rich-get-richer hypothesis assumes that particularly adolescents with a high level of selfesteem experience SM-induced increases in self-esteem, which come on top of the many benefits that these adolescents already experience in their offline lives (Kraut et al., 2002; Valkenburg \& Peter, 2011). Conversely, a social compensation hypothesis assumes that particularly adolescents with a low level of self-esteem experience SM-induced increases in their self-esteem, which may compensate for the lack of positive experiences in their offline lives (Kraut et al., 2002; Valkenburg \& Peter, 2011).

While the rich-get-richer and social compensation hypotheses are based solely on self-esteem level (whether it is high or low), selfesteem instability theory (Kernis, 2005) argues that it is not so much self-esteem level but self-esteem instability that may inform hypotheses on the influence of the valence of experiences on self-esteem. For example, Kernis (2005) argues that the individuals with instable high self-esteem do show susceptibility to negative experiences, but those with stable high self-esteem do not. Hence, it is not only selfesteem level, but also self-esteem instability that may explain how adolescents respond to SM. Based on these insights, we investigated whether the within-person effects of time spent on SM (RQ2a) and the valence of SM experiences (RQ2b) on self-esteem depend on adolescents' self-esteem level and self-esteem instability.

\section{Peer Approval and Physical Appearance Contingencies}

Self-esteem contingencies are the unique domains of life that serve as the basis of our self-esteem (Crocker et al., 2003). Having contingent self-esteem is functional for cognitive and psychosocial development. After all, if our self-esteem remains stable, no matter what happens, there is no motivation to learn and develop (Vonk \& Smit, 2012). Adolescents do seem to differ, though, in the domains on which their self-esteem is contingent. For example, whereas 
some adolescents' self-esteem may particularly depend on academic performance, others may base their self-esteem on peer approval.

Different contexts may also activate different self-esteem contingencies (Crocker \& Brummelman, 2018). In the classroom, academic competence is valued, which may activate the academic competence contingency. In SM interactions, peer approval and physical appearance are relevant, so that these self-esteem contingencies may particularly be triggered in the SM context. It is, therefore, conceivable that adolescents who particularly base their self-esteem on peer approval or physical appearance are more susceptible to the effects of time spent on SM and the valence of $\mathrm{SM}$ experiences on self-esteem than adolescents who do less so. Therefore, we investigated whether the within-person effects of time spent on SM (RQ3a) and the valence of SM experiences (RQ3b) on self-esteem depend on adolescents' peer approval and physical appearance contingencies.

\section{Method}

This preregistered (https://osf.io/43m7t) study was part of a larger longitudinal project on the psychosocial consequences of SM use among middle adolescents (13-16 years) that ran from November 2019 to June 2020 (see our website). This larger project consists of a measurement burst design. Such a design, which was first described by Nesselroade (1991), combines two types of data: (a) longitudinal data with widely spaced intervals (e.g., months, years) and (b) longitudinal data with closely spaced intervals (e.g., hours, days), which are, for example, obtained via ESM or daily dairy studies. These second types of data are called measurement "bursts" (Nesselroade, 1991, p. 235).

In our larger project, adolescents completed 16 biweekly online surveys and two 3-week pre-ESM surveys, and participated in two 3-week ESM bursts, one in November/December 2019 and one in June 2020. The design and sampling plan (https://osf.io/327cx) of the larger project was preregistered in August 2019 before recruiting participants and collecting data. The hypotheses and analysis plan (https://osf.io/43m7t) for the present study was preregistered in October 2020, before analyzing the data of this study.

The present study was based on data from the second ESM study (June 2020), the second pre-ESM survey, and two biweekly surveys, fielded in the first week and just after the third week of this ESM study. The present study built upon Valkenburg, Beyens, et al. (2021a), which was based on the first ESM study, in three respects. First, it aimed to replicate Valkenburg, Beyens, et al.'s findings on the associations of time spent using SM with self-esteem, using a different measure of time spent with SM. Second, it extended Valkenburg, Beyens, et al.'s study by including the valence of SM experiences as a comparative predictor of self-esteem. Third, it investigated the role of five moderators that may explain the heterogeneity in the person-specific effects on self-esteem.

\section{Participants}

The final sample of this study consisted of 300 adolescents (58\% girls; $M_{\text {age }}=14.61, S D=.70$ ) from Grades 8 and 9 of a large secondary school in the south of the Netherlands. Adolescents were enrolled in different educational tracks: $37 \%$ were in lower prevocational secondary education, $34 \%$ in intermediate general secondary education, and $29 \%$ in academic preparatory education. Of all adolescents, 97\% were born in the Netherlands and 98\% identified themselves as Dutch. The sample was a fairly accurate representation of the specific area in the Netherlands in terms of educational level and ethnic background.

A priori power analyses using Monte Carlo simulations (https:// osf.io/ar4vm/) indicated that a sample size of 300 participants (with 42 assessments) would be sufficient to reliably detect small withinperson and moderator effects with a power of .80 and significance level of .05. The original sample of the larger study consisted of 388 adolescents who provided informed consent themselves. Of these 388 adolescents, 300 participants (77\%) who used WhatsApp, Instagram, or Snapchat participated in the second ESM wave.

\section{Procedure}

The study was approved by the Ethics Review Board of the University of Amsterdam. Before the start of the study, parents gave written consent for their child's participation in the study. Two weeks before the start of the larger project in November 2019, adolescents participated in a classroom session in which they were informed extensively about the aims and procedures of the study. Two weeks before the start of the second ESM study, adolescents received an online instruction on how to install the ESM software application (Ethica Data) on their phones. At this time, adolescents also completed a pre-ESM survey via the Ethica Data app to investigate their use of different SM platforms.

\section{ESM Surveys}

Adolescents received six 2-min surveys per day via their mobile phones (Ehtica Data software). Each survey consisted of 19-32 questions, depending on the moment of the day. All surveys included questions on adolescents' self-esteem, time spent on $\mathrm{SM}$, and the valence (positivity or negativity) of their SM experiences. Adolescents only received questions about their time spent on Instagram, WhatsApp, and Snapchat if they had indicated in the preESM survey that they used these platforms more than once a week. In total, 293 (98\%) adolescents received questions about WhatsApp, 261 adolescents (87\%) about Instagram, and 232 (77\%) about Snapchat.

Sampling Scheme/Monitoring Plan. Adolescents received a total of 126 ESM surveys (i.e., 21 days $* 6$ assessments per day) at random time points within fixed intervals. A detailed overview of the notification scheme with the response windows can be found on OSF (http://osf.io/vkr4u). We messaged adolescents daily to motivate them to fill out as many ESM surveys as possible and to check whether we could help with any technical issues. If adolescents did not complete an ESM survey within $10 \mathrm{~min}$, they received an automatic reminder via the Ethica Data app. Adolescents received a compensation of $€ 0.30$ for each completed ESM survey and an additional compensation of $€ 0.20$ if they completed the final (longer) ESM survey of the day. In addition, each day, we organized a lottery, in which four adolescents who had completed all six ESM surveys on the previous day could win $€ 25$.

Compliance. Due to unforeseen technical problems with the Ethica software, 140 ESM surveys (0.37\%) of the 37,800 surveys that were sent out were not received by adolescents. As a result, adolescents could have completed a maximum number of 37,660 ESM surveys, of which they actually completed (or partially 
completed) 21,970 surveys. This resulted in a compliance rate of $58 \%$, which is reasonable (van Roekel et al., 2019). On average, adolescents completed 73.23 ESM surveys $(S D=34.77)$.

\section{Online Surveys}

On the first day of the current ESM study, adolescents received a link to a 5-min survey. This survey contained, among other instruments, items about the two self-esteem contingencies. A small percentage of adolescents $(9 \%)$ did not complete this survey within 2 weeks. To prevent missing cases (and thus reduced statistical power), these adolescents were asked to complete the self-esteem contingencies questions as part of the end-of-study survey, which was fielded just after the end of the ESM study. Adolescents received a compensation of $€ 2$ for completing each online survey. Moreover, all adolescents who completed the online survey within 2 days participated in an additional lottery in which four of them could win $€ 25$.

\section{Measures \\ Self-Esteem (ESM)}

Based on research that established the validity of single-item measures of self-esteem (e.g., Robins et al., 2001), we measured adolescents' self-esteem by asking participants "How satisfied about yourself do you feel right now?." Adolescents answered on a 7-point scale, ranging from 0 (not at all) to 6 (completely), with 3 (a little) as the midpoint. Adolescents' self-esteem level was inferred from the latent mean of all 126 self-esteem observations. Self-esteem instability was computed by calculating the intraindividual standard deviation of self-esteem across all ESM assessments (cf., Meier et al., 2011).

\section{Time Spent on SM (ESM)}

We asked adolescents how much time in the past hour they had spent with the three most popular platforms in this age group: WhatsApp, Instagram, and Snapchat. Response options ranged from $0 \mathrm{~min}$ to $60 \mathrm{~min}$, with 1-min intervals. Adolescents' time scores for each of the three platforms were summed. In $2.7 \%$ of the observations, the estimated time exceeded $60 \mathrm{~min}$. In accordance with our preregistration, these observations were recoded to $60 \mathrm{~min}$.

\section{Valence of Experiences While Using SM (ESM)}

Estimating the valence of social media experiences among middle adolescents is a challenge because middle adolescents often have a hard time understanding certain terms that are commonly used in scales designed for older adolescents or adults. Based on pilot interviews, we learned that a positive experience is an experience they like. If adolescents indicated that they had spent at least $1 \mathrm{~min}$ using WhatsApp, Instagram, or Snapchat in the past hour, we asked them: "How much did you like your experience on social media in the past hour?." Response options ranged from 0 (not at all) to 6 (very much), with 3 (a little) as the midpoint.

\section{Peer Approval and Appearance Contingencies of Self-Esteem (Survey)}

To measure the peer approval contingency, we presented adolescents with the following two statements: "I feel more satisfied about myself ... (a) when others praise me and (b) when I get a lot of attention from others." To measure the appearance contingency, we used the following two statements: "I feel more satisfied about myself ... (a) when I think I am looking good and (b) when I think I am attractive." Response options ranged from 0 (do not agree at all) to 4 (completely agree). Following the suggestion of the editor, we deviated from our preregistration and conducted a Confirmatory Factor Analysis rather than a Principal Component Analysis with two fixed factors. The first factor represented the appearance items (Cronbach's $\alpha=.82$; Spearman's rho $=.70$ ), the second factor represented the peer approval items (Cronbach's $\alpha=.63$; Spearman's rho $=.40)$. The intercorrelation of the two subscales was $r=.55(p<.001)$. For loadings and fit indices, see OSF Supplemental 1 (https://osf.io/cn3xd/).

\section{Method of Analysis}

We employed DSEM for intensive longitudinal data in Mplus Version 8.4 (Asparouhov et al., 2018). Following our preregistration (https://osf.io/43m7t) we estimated two separate two-level autoregressive lag-1 models, one for time spent on SM, and one for the valence of SM experiences. A detailed overview of the model specifications and overall model fits can be found in OSF Supplemental 2 (https://osf.io/knsv6/). At the within-person level (Level 1) of both models, we controlled for the autoregressive effect of selfesteem (i.e., self-esteem predicted by self-esteem at the previous measurement). In the first model, we included time spent on SM in the past hour as the time-varying covariate (no hypothesis, Model 1). In the second model, we included the valence of SM experiences in the past hour as the time-varying covariate (H4, Model 2). As suggested by Adachi and Willoughby (2015), we considered an effect size of $\beta=.05$ as the smallest effect size of interest for the within-person effects of time spent with SM and the valence of SM experiences on self-esteem.

At the between-person level (Level 2), we examined correlations between the latent mean of self-esteem and the latent mean of time spent on SM (H1, Model 1) and the latent mean of the valence of SM experiences (H3, Model 2). In addition, we investigated heterogeneity in the within-person effects (i.e., random effects), by specifying the between-person variances around the within-person effects of time spent with SM on self-esteem (H2, Model 1) and the valence of SM experiences on self-esteem (H5, Model 2). Finally, we investigated how each of the five moderators (i.e., gender, selfesteem level, self-esteem instability, and the two self-esteem contingencies) were associated with the person-specific within-person effects (variance around Beta) of time spent with SM (RQ1a, RQ2a, RQ3a; Model 1) and the valence of SM experiences on self-esteem (RQ1b, RQ2b, RQ3b; Model 2).

\section{Results}

\section{Descriptives and Correlations}

Table 1 presents the number of observations, range, means, standard deviations, within-person, and between-person correlations of all 
Table 1

Descriptives, Within-Person, and Between-Person Correlations Between Study Variables

\begin{tabular}{|c|c|c|c|c|c|c|c|c|c|c|}
\hline \multirow[b]{2}{*}{ Study variable } & \multicolumn{4}{|c|}{ Descriptives $^{\mathrm{a}}$} & \multicolumn{6}{|c|}{ Correlations $^{\mathrm{b}}$} \\
\hline & Obs & Range & $M$ & $S D$ & 1 & 2 & 3 & 4 & 5 & 6 \\
\hline 1. Self-esteem & 21,906 & $0-6$ & 4.16 & 1.06 & - & $-.02 * * *$ & $.18^{* * *}$ & & & \\
\hline 2. T-SM & 21,858 & $0-60$ & 14.79 & 11.98 & $-.14^{*}$ & - & .01 & & & \\
\hline 3. V-SM & 15,708 & $0-6$ & 3.77 & 1.22 & $.55^{* * *}$ & $-.16^{* *}$ & - & & & \\
\hline 4. Gender $(0=$ boys $)$ & 299 & $0-1$ & 0.58 & 0.49 & -.06 & .10 & $.21^{* * *}$ & - & & \\
\hline 5. Self-esteem instability & 300 & $0-2.74$ & 0.90 & 0.45 & $-.27^{* * *}$ & .07 & $-.33^{* * *}$ & -.06 & - & \\
\hline 6. SEC-Approval & 287 & $0-4$ & 2.49 & 0.95 & $.19^{* * *}$ & .09 & .11 & .09 & .04 & - \\
\hline 7. SEC-Appearance & 287 & $0-4$ & 2.15 & 1.10 & .04 & $.13^{*}$ & .06 & $.23^{* * *}$ & -.01 & $.55^{* * *}$ \\
\hline
\end{tabular}

Note. $\quad$ Obs $=$ total number of observations; T-SM = time spent on social media; V-SM = valence of social media experiences; SEC = self-esteem contingency. ${ }^{a}$ Means of self-esteem, time spent on SM, and valence of SM experiences represent the average of the person-mean scores. ${ }^{\mathrm{b}}$ Within-person correlations are presented above and between-person correlations below the diagonal.

${ }^{*} p \leq .05$. ** $p \leq .01$. *** $p \leq .001$.

variables in the study. As the Table shows, we replicated the high average level of self-esteem of adolescents that was found by Valkenburg, Beyens, et al. (2021a). Adolescents spent on average almost 15 min using SM in the hour before each observation. Furthermore, their experiences with SM across the 3 weeks were more positive than negative $(M=3.77, S D=1.22$, range $=0-6)$. Across 15,708 ESM observations of the valence of SM experiences, $55 \%$ of adolescents' experiences were positive $(\geq 4)$, whereas $18 \%$ of their experiences were negative $(\leq 2)$. The remaining $27 \%$ of the experiences were scored on the midpoint of the scale (3). Finally (not reported in Table 1), the intraclass correlations (ICCs) were .53 for self-esteem, .51 for time spent with SM, and .50 for the valence of SM experiences.

\section{Investigating Hypotheses and Research Questions for Time Spent With SM}

The outcomes of the model analyzing the effects of time spent with SM on self-esteem are included in Table 2. In support of our first hypothesis (H1) and consistent with Valkenburg, Beyens, et al. (2021a), we found that, overall, adolescents who spent more time on social media had lower levels of self-esteem than adolescents who spent less time on social media (i.e., negative between-person association; $\beta=-.14 ; p=.01)$. The overall within-person effect of time spent with SM on self-esteem while controlling for selfesteem at the previous assessment was nonsignificant $(\beta=-.01$; $p=.08)$. However, in support of our second hypothesis $(\mathrm{H} 2)$, we

Table 2

Main Outcomes Dynamic Structural Equation Modeling Model of Time Spent on Social Media and Self-Esteem

\begin{tabular}{|c|c|c|c|c|}
\hline Fixed effects and associations & $b$ & $\beta$ & $p$ & $95 \% \mathrm{CI}$ \\
\hline \multicolumn{5}{|l|}{ Fixed effects } \\
\hline \multicolumn{5}{|l|}{ Within-person effects } \\
\hline T-SM $\rightarrow$ S-E (Beta) & -.013 & -.013 & .081 & {$[-.030, .004]$} \\
\hline $\mathrm{S}-\mathrm{E}(t-1) \rightarrow \mathrm{S}-\mathrm{E}(t, \mathrm{Phi})$ & .195 & .195 & .000 & {$[.170, .219]$} \\
\hline \multicolumn{5}{|l|}{ Between-person associations } \\
\hline T-SM and S-E (H1) & -.180 & -.141 & .011 & {$[-.256,-.023]$} \\
\hline Beta and gender $(0=$ boys, RQ1a) & .002 & -.057 & .166 & {$[-.175, .057]$} \\
\hline Beta and S-E level (RQ2a) & -.027 & -.232 & .006 & {$[-.412,-.051]$} \\
\hline Beta and S-E instability (RQ2a) & -.012 & -.248 & .007 & {$[-.425,-.050]$} \\
\hline Beta and SEC: Approval (RQ3a) & -.009 & -.086 & .161 & {$[-.256, .087]$} \\
\hline Beta and SEC: Appearance (RQ3a) & .006 & .046 & .303 & {$[-.138, .230]$} \\
\hline Random effects and variances & $\sigma^{2}$ & & $p$ & $95 \% \mathrm{CI}$ \\
\hline \multicolumn{5}{|l|}{ Random effects } \\
\hline \multicolumn{5}{|l|}{ Random slopes } \\
\hline T-SM $\rightarrow$ S-E (H2, Beta) & 0.012 & & .000 & {$[.008, .018]^{\mathrm{a}}$} \\
\hline $\mathrm{S}-\mathrm{E}(t-1) \rightarrow \mathrm{S}-\mathrm{E}(t, \mathrm{Phi})$ & 0.063 & & .000 & {$[.050, .079]$} \\
\hline \multicolumn{5}{|l|}{ Variances predictor and outcome } \\
\hline T-SM (within-person) & 1.283 & & .000 & {$[1.259,1.307]$} \\
\hline T-SM (between-person) & 1.412 & & .000 & {$[1.196,1.682]$} \\
\hline S-E (within-person, residual) & 0.840 & & .000 & {$[.823, .857]$} \\
\hline S-E (between-person) & 1.159 & & .000 & {$[.986,1.389]$} \\
\hline
\end{tabular}

Note. $\quad b s$ are unstandardized. Betas ( $\beta \mathrm{s})$ are standardized using the STDYX Standardization in Mplus. $p$ values are one-tailed Bayesian $p$ values (McNeish \& Hamaker, 2020). Significant fixed effects are depicted in bold. DSEM = dynamic structural equation modeling; T-SM = time spent on social media; S$\mathrm{E}=$ self-esteem; $\mathrm{SEC}=$ self-esteem contingency; $\mathrm{RQ}=$ research question; $\mathrm{H}=$ hypothesis .

${ }^{a}$ The 95\% Credible Interval of the variance around the effect of T-SM on S-E (Beta) indicates that the within-person effect of T-SM on S-E differed among participants. 
did find significant heterogeneity in this overall within-person effect (random effect $=0.012, p=.000$ ), with $N=1$ effect sizes of time spent with SM on self-esteem ranging from $\beta=-.31$ to $\beta=+.27$ across adolescents. When expressed in percentages, $56 \%$ of adolescents experienced no to very small effects of time spent with SM on self-esteem $(-.05<\beta<.05)$, whereas $27 \%$ experienced negative effects $(\beta \leq .05)$, and $18 \%$ positive effects $(\beta \geq .05)$. These effect sizes are visualized in the bottom left histogram in Figure 1.

Finally, we examined whether the strength and direction of the person-specific within-person effects of time spent with SM on selfesteem (i.e., Beta) depended on gender (RQ1a), self-esteem level, self-esteem instability (RQ2a), and the peer approval and physical appearance self-esteem contingencies (RQ3a). We found evidence for a moderator effect of self-esteem level $(\beta=-.23, p=.01)$ and self-esteem instability $(\beta=-.25, p=.01)$, but not of gender and the two self-esteem contingencies. The two significant moderator effects indicated that, compared to their peers, adolescents with a lower self-esteem level (see top left histogram in Figure 1) and a lower self-esteem instability (see bottom second left histogram in Figure 1) experienced increases in self-esteem after spending more time with SM. Conversely, more adolescents with a higher selfesteem level (see top right histogram in Figure 1) and a higher selfesteem instability (see bottom right histogram Figure 1) experienced decreases in self-esteem after spending more time with SM.

\section{Investigating Hypotheses and Research Questions for the Valence of SM Experiences}

The outcomes of the model analyzing the effects of the valence of SM experiences on self-esteem are presented in Table 3. In support of our third hypothesis (H3), we found that, overall, adolescents who had more positive SM experiences (i.e., valence) had higher levels of self-esteem than adolescents with less positive SM experiences (i.e., positive between-person association; $\beta=.57 ; p=.000)$. In support of our fourth hypothesis (H4), we also found a significant positive overall within-person effect of the valence of SM experiences on self-esteem $(\beta=.15 ; p=.000)$. This finding indicates that adolescents' self-esteem increased (compared to their average self-esteem level) when their SM experiences in the previous hour were more positive. And finally, in support of our H5, we found significant heterogeneity in this within-person effect (random effect $=0.032, p=.000$ ). The person-specific effects of the valence of SM experiences on self-esteem ranged from $\beta=-.45$ to $\beta=+.59$. Expressed in percentages, $78 \%$ of adolescents experienced a positive effect of the valence of SM experiences on self-esteem $(\beta \geq .05)$, while $19 \%$ experienced no to a very small effect $(-.05<\beta<.05)$, and $3 \%$ a negative effect $(\beta \leq .05)$. The range of these person-specific effect sizes is visualized in the bottom left-hand histogram in Figure 2 .

\section{Figure 1}

The Ranges of the Person-Specific Effects of Time Spent With Social Media (SM) on Self-Esteem for All Adolescents, and for Those With Low, Average, and High Self-Esteem Level and Self-Esteem Instability

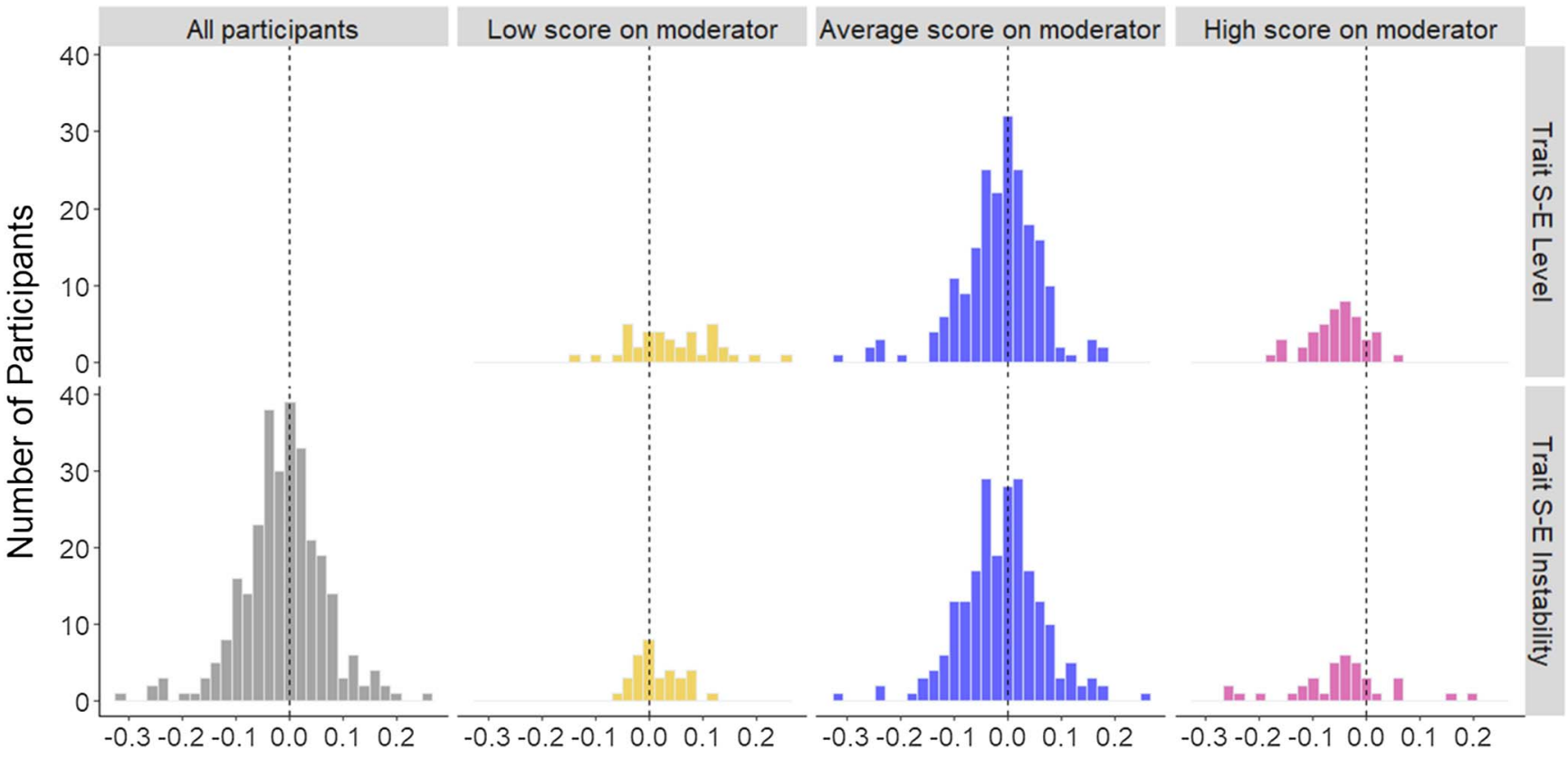

Note. The $x$-axis represents the person-specific effect sizes of time spent with SM on self-esteem (Betas), which ranged from $\beta=-.307$ to $\beta=+.266$ across all 300 adolescents (see bottom left plot). The upper three plots show the person-specific effects of time spent with SM on self-esteem for adolescents with low ( $<1 S D$ of the mean), average (within $1 S D$ of the mean), and high ( $>1 S D$ of the mean) self-esteem levels. Although all three upper plots show sizeable heterogeneity in effects within each subgroup, adolescents with a low self-esteem level tended to experience more positive effects of time spent with SM on selfesteem, whereas adolescents with average or high levels of self-esteem tended to experience more negative within-person effects. The lower three right plots show the person-specific effects of time spent with SM on self-esteem for adolescents with low, average, and high self-esteem instability. Although these plots also show sizeable heterogeneity within these subgroups, adolescents with high self-esteem instability tended to experience more negative effects of time spent using SM on self-esteem, whereas adolescents with average or low self-esteem instability tended to experience more positive effects. 
Table 3

Main Outcomes Dynamic Structural Equation Modeling Model of the Valence of Social Media Experiennces and Self-Esteem

\begin{tabular}{|c|c|c|c|c|}
\hline Fixed effects and associations & $b$ & $\beta$ & $p$ & $95 \% \mathrm{CI}$ \\
\hline \multicolumn{5}{|l|}{ Fixed effects } \\
\hline \multicolumn{5}{|l|}{ Within-person effects } \\
\hline V-SM $\rightarrow$ S-E (Beta, H4) & 0.131 & 151 & .000 & {$[.125, .172]$} \\
\hline S-E $(t-1) \rightarrow$ S-E $(t$, Phi $)$ & 0.185 & .189 & .000 & {$[.161, .219]$} \\
\hline \multicolumn{5}{|l|}{ Between-person associations } \\
\hline V-SM and S-E (H3) & 0.764 & .569 & .000 & {$[.510, .673]$} \\
\hline Beta and gender $(0=$ boys; RQ1b $)$ & 0.007 & .082 & .138 & {$[-.068, .231]$} \\
\hline Beta and S-E level (RQ2b) & 0.014 & .075 & .188 & {$[-.087, .233]$} \\
\hline Beta and S-E instability (RQ2b) & 0.029 & .349 & .000 & {$[.191, .493]$} \\
\hline Beta and SEC: Approval (RQ3b) & 0.035 & .202 & .006 & {$[.051, .346]$} \\
\hline Beta and SEC: Appearance (RQ3b) & 0.017 & .086 & .131 & {$[-.067, .238]$} \\
\hline Random effects and variances & $\sigma^{2}$ & & $p$ & $95 \% \mathrm{CI}$ \\
\hline \multicolumn{5}{|l|}{ Random effects } \\
\hline \multicolumn{5}{|l|}{ Random slopes } \\
\hline V-SM $\rightarrow$ S-E (H5, Beta) & 0.032 & & .000 & {$[.024, .042]^{\mathrm{a}}$} \\
\hline $\mathrm{S}-\mathrm{E}(t-1) \rightarrow \mathrm{S}-\mathrm{E}(t, \mathrm{Phi})$ & 0.088 & & .000 & {$[.070, .109]$} \\
\hline \multicolumn{5}{|l|}{ Variances predictor and outcome } \\
\hline V-SM (within-person) & 1.430 & & .000 & {$[1.398,1.462]$} \\
\hline V-SM (between-person) & 1.499 & & .000 & {$[1.274,1.784]$} \\
\hline S-E (within-person, residual) & 0.747 & & .000 & {$[.729, .765]$} \\
\hline S-E (between-person) & 1.102 & & .000 & {$[.933,1.311]$} \\
\hline
\end{tabular}

Note. $\quad b \mathrm{~s}$ are unstandardized. Betas $(\beta \mathrm{s})$ are standardized using the STDYX Standardization in Mplus. $p$ values are one-tailed Bayesian $p$ values (McNeish \& Hamaker, 2020). Significant fixed effects are depicted in bold. The analyses were based on a subset of 15,708 observations on which adolescents spent time on Instagram, WhatsApp, or Snapchat. DSEM = dynamic structural equation modeling; V-SM = valence of social media experiences; S-E = self-esteem; $\mathrm{SEC}=$ self-esteem contingency; $\mathrm{RQ}=$ research question; $\mathrm{H}=$ hypothesis .

${ }^{a}$ The 95\% Credible Interval of the variance around the effect of V-SM on S-E (Beta) indicates that the within-person effect of V-SM on S-E differed among participants.

Finally, we examined whether the strength and direction of the person-specific within-person effects of the valence of SM experiences on self-esteem (i.e., Beta) depended on gender (RQ1b), selfesteem level, self-esteem instability (RQ2b), and the peer approval and physical appearance self-esteem contingencies (RQ3b). We found evidence for moderator effects of self-esteem instability $(\beta=.35, p=.00)$ and the peer approval contingency $(\beta=.20$, $p=.006)$, but not of gender, self-esteem level, and the physical appearance contingency. The two significant moderator effects indicated that adolescents with a higher self-esteem instability (see bottom right histogram in Figure 2), as well as adolescents with a higher peer approval contingency (see top right histogram in Figure 2) experienced stronger increases in self-esteem due to positive SM experiences than adolescents with a lower self-esteem instability (see top left histogram in Figure 2) and a lower peer approval contingency (see bottom second left histogram Figure 2).

\section{Exploratory and Sensitivity Analyses}

As preregistered, we conducted a validation check to examine whether findings were robust against outliers and potential untrustworthy answer patterns, which they were (for results, see OSF Supplemental 3, https://osf.io/u7deq/). We also conducted analyses with time spent using SM and the valence of SM experiences included as predictors in the same model (instead of in two separate models). This model seemed to be too complex because even after 50,000 iterations it did not converge (see OSF Supplemental 4, https://osf.io/t35hd/). Finally, because the null effects for the moderator gender were consistent with the results of earlier studies, we investigated to what extent the person-specific effects of time spent with SM and the valence of SM experiences on self-esteem differed within each of the two gender groups. As the histograms show (see OSF Supplemental 5, https://osf.io/uyxrw/), the ranges of the person-specific effect sizes were highly comparable among boys and girls. Finally, following a suggestion of one of the reviewers, we investigated whether the effects of time spent on SM and the valence of SM experiences on self-esteem were moderated by adolescents' educational level. Results showed no significant moderating effect of educational level for time spent on SM, $\beta=-.12$, CI [-.228; $.002]$, and the valence of SM experiences, $\beta=.05$, CI [-.105; .204].

\section{Discussion}

The first aim of this ESM study was to investigate and replicate the effect of time spent with SM on adolescents' self-esteem reported by Valkenburg, Beyens et al. (2021a), and to compare this effect with that of the valence of adolescents' SM experiences. Consistent with sociometer theory (Leary \& Baumeister, 2000), our results revealed that the valence (the positivity and negativity) of adolescents' SM experiences was a more important predictor of surges and drops in self-esteem than time spent with SM.

\section{Between-Person Associations of Social Media Use With Self-Esteem}

Consistent with H1 and earlier studies (e.g., Rodgers et al., 2020; Valkenburg, Beyens, et al., 2021a), we found a negative betweenperson relation between time spent on SM and self-esteem 
Figure 2

The Ranges of the Person-Specific Effects of the Valence of Social Media (SM) Experiences on Self-Esteem for All Adolescents, and for Those With Low, Average, and High Peer Approval Contingency and Self-Esteem Instability

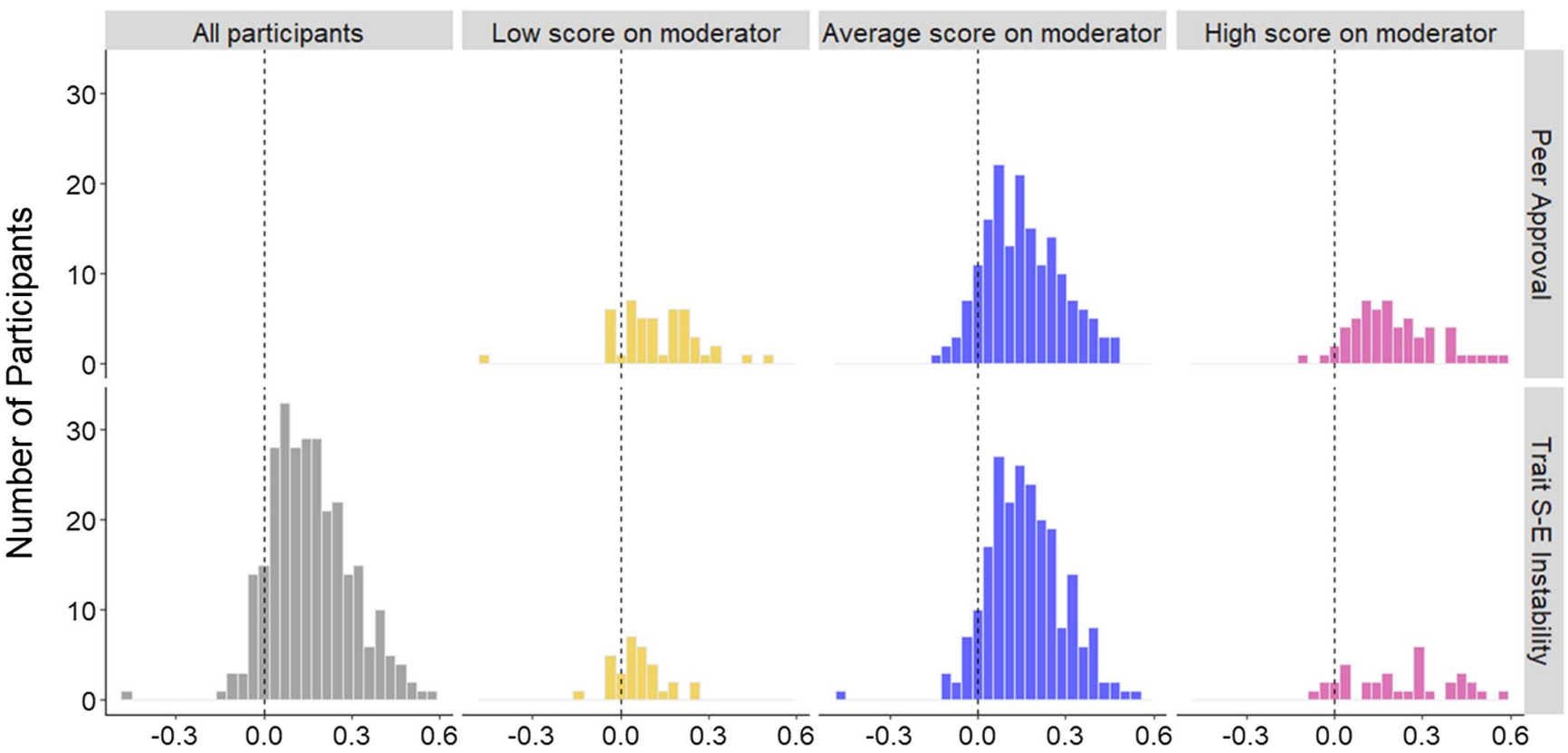

Note. The $x$-axis represents the person-specific effect sizes of the valence of SM experiences on self-esteem (Betas), which ranged from $\beta=-.453$ to $\beta=+.588$ across all 300 adolescents (see bottom left plot). The upper three plots show the person-specific effects of the valence of SM experiences on selfesteem for adolescents with low ( $<1 S D$ of the mean), average (within $1 S D$ of the mean), and high peer approval contingency ( $>1 S D$ of the mean). Although these three plots all show a strong tendency toward positive effects of the valence of their SM experiences, adolescents with a high peer approval contingency tended to experience even stronger positive effects of the valence of their SM experiences on self-esteem than adolescents with average and low levels of peer approval contingency. The three bottom right histograms show the person-specific effects of the valence of SM experiences for adolescents with low, average, and high self-esteem instability. Although all these three plots show trends toward positive effects, adolescents with high self-esteem instability tended to experience even stronger positive effects of positive SM experiences on self-esteem than adolescents in the low and average groups.

$(r=-.14)$. But in support of $\mathrm{H} 3$, we found a strong positive between-person association of the valence of SM experiences with self-esteem $(r=+.57)$. These opposite relations align with the results of a meta-analysis of Liu and Baumeister (2016), which found that quantitative measures, such as time spent on SM, resulted in negative between-person relations with self-esteem, while more qualitative measures, such as interactions with friends on SM, yielded positive between-person relations with self-esteem. Our between-person associations suggest that adolescents with lower self-esteem levels than their peers spend more time on SM and have fewer positive experiences on SM.

\section{Within-Person Associations of Social Media Use With Self-Esteem}

We also found sizeable differences in the predictive power of time spent on SM and the valence of SM experiences on self-esteem. While the overall within-person effect of time spent on SM was close to zero, which replicated the finding of Valkenburg, Beyens, et al. (2021a), the within-person effect of the valence of SM experiences was positive. This latter effect meant that, consistent with sociometer theory (Leary \& Baumeister, 2000), adolescents' self-esteem surged after positive SM experiences, whereas it dropped after negative SM experiences.
However, these momentary ups and downs in self-esteem must be seen in light of the balance between positive and negative SM experiences among adolescents. Our results showed that $55 \%$ of all observed SM experiences were positively valenced, whereas $18 \%$ were negatively valenced, a result that is consistent with earlier studies reporting a sizeable positivity bias in SM interactions (e.g., Primack et al., 2019; Reinecke \& Trepte, 2014; Waterloo et al., 2018). However, if adolescents' experiences were more positive than negative, they also experienced more ups than downs in selfesteem across the 3-week period. And this means, consequently and reassuringly, that the significant positive within-person effect of the valence of adolescents' SM experiences is more due to the ups in self-esteem after positive SM experiences than to the downs in selfesteem after negative SM experiences.

In support of $\mathrm{H} 2$ and $\mathrm{H} 5$, time spent with $\mathrm{SM}$ and the valence of SM experiences led to sizeable heterogeneity in person-specific effects across adolescents. In accordance with media effects theories (Valkenburg \& Peter, 2013) and self-esteem theories (Harter, 2012), adolescents differed substantially in their susceptibility to the effects of SM use on self-esteem. A comparison of Figures 1 and 2 shows that the person-specific effects of time spent with SM on self-esteem centered around the overall within-person effect of $\beta=.01$ (ranging from $\beta=-.31$ to $\beta=+.27$ ), whereas the person-specific effects of the valence of SM experiences on self-esteem concentrated at the 
right side of the histogram around the overall within-person effect of $\beta=.15$ (ranging from $\beta=-.45$ to $\beta=+.59$ ).

\section{Explaining Person-Specific Effects of SM Use on Self-Esteem}

The second aim of this study was to examine the moderating role of five moderators of the effects of SM use on self-esteem: gender, self-esteem level and instability, and peer approval and physical appearance contingencies. The explanatory power of these moderators varied considerably. First, consistent with earlier betweenperson studies (e.g., Meeus et al., 2019), gender did not moderate the effects of time spent on SM and those of the valence of SM experiences on self-esteem (RQ1a/b). As OSF Supplemental 5 (https://osf.io/uyxrw/) shows, the person-specific effects of both time spent on SM and the valence of SM experiences ranged just as strongly within the boys' and girls' groups as they did across these groups. The lack of moderation by gender found in our and earlier studies is apparently due to the high heterogeneity within each of the gender groups, which may have hampered the detection of differences between these groups.

The differences in person-specific effect sizes could be explained by self-esteem level and self-esteem instability (RQ2a/b). First, adolescents with a lower average self-esteem experienced stronger positive effects of time spent with SM on self-esteem than adolescents with higher average self-esteem. This result may point at a social compensation effect (Kraut et al., 2002), indicating that especially adolescents with a low self-esteem use SM to boost their self-esteem. However, as argued in self-esteem theories, probably due to self-protective processes, hardly any adolescent reports a stable low level of self-esteem (Kernis, 2003; cf., Valkenburg, Beyens, et al., 2021a), which also applies to the present study. Self-esteem theories also argue that it is not so much self-esteem level but self-esteem instability that may inform hypotheses on the influence of one's experiences on self-esteem (e.g., Kernis, 2003).

Regarding self-esteem instability, we found that especially adolescents with high self-esteem instability tended to experience negative effects of time spent on SM on self-esteem, which is at odds with the social compensation hypothesis. For these adolescents, we also found a stronger positive effect of the valence of SM experiences on self-esteem, which may, at first sight, also be at odds with the social compensation hypothesis. However, this latter result must be seen in light of our finding that adolescents with a high selfesteem instability reported significantly fewer positive experiences on SM than their peers (see Table 1). Therefore, for these adolescents, the positive effect of the valence of SM experiences on selfesteem may pertain more to drops in self-esteem as a result of negative experiences on SM than to surges in self-esteem as a result of positive experiences on SM. Finally, since self-esteem instability explained only a part of the effect of the valence of SM experiences on self-esteem, other moderators may have played a role. For example, it is possible that adolescents with high self-esteem instability also more often experienced negative offline situations than their peers, and that these negative offline experiences may have co-affected their self-esteem. In all, self-esteem instability seems to be a critical susceptibility factor to explain the momentary effects of positive and negative SM experiences on self-esteem. This result is in line with recent studies showing that adolescents with high mood instability are more prone to develop depressive symptoms than peers with more stable moods (e.g., Maciejewski et al., 2019).

Finally, differences in the person-specific effects of the time spent with SM and the valence of SM experiences on self-esteem could partly be explained by adolescents' peer approval contingency, but not by their physical appearance contingency (RQ3a/b). First, high peer approval or physical appearance contingency did not result in more sizeable person-specific effects of time spent with SM on selfesteem. However, adolescents who particularly based their selfesteem on peer approval did show more susceptibility to the effects of positively valenced SM experiences on their self-esteem than adolescents who did less so. It is conceivable that these adolescents are particularly focused on SM interactions that, for example, elicit positive feedback from their peers to boost their self-esteem.

\section{Avenues for Future Research}

Our study also made a first step in investigating potentially valid moderators to explain differences in the person-specific susceptibilities of SM use on self-esteem. We focused mostly on dispositional moderators, such as self-esteem instability and self-esteem contingencies. However, although important as a first step, both developmental and media effect theories argue that the effects of SM use on self-esteem depend on a unique combination of dispositional, developmental, and social-context factors that may differ from adolescent to adolescent (e.g., Bronfenbrenner, 2005; Valkenburg \& Peter, 2013). Future research may therefore extend our study by focusing on other potential dispositional moderators, such as social comparison orientation or social anxiety, in addition to developmental and social-context moderators, such as the norms in families or peer groups. Although this study clearly showed that each and every adolescent may respond differently to SM use, only by investigating the combined validity of dispositional, developmental, and social-context moderators can we truly understand why SM use leads to positive effects among some adolescents, negative effects among others, and null effects among yet others.

Future research may also extend our findings, for example by including more fine-grained measures of SM use than overall time spent on SM as we did in the present study. Several recent survey and ESM studies have investigated the differential effects of passive and active SM use (Beyens et al., 2020; Escobar-Viera et al., 2018). To our knowledge, the passive and active SM use dichotomy was introduced by Burke et al. (2010), and it sparked dozens of subsequent survey and ESM studies (Valkenburg, Beyens, et al., 2021b). However, studies into the effects of active and passive SM use still only focus on time spent on social media, albeit time spent browsing and posting. What we really need are studies to investigate the effects of the specific content of adolescents' SM interactions on their self-esteem.

Investigating the content of SM interactions in survey studies is difficult if not impossible. But it can be realized by linking survey data to additional data collection methods, such as random screenshots of SM interactions (Reeves et al., 2021) or SM data downloads (Boeschoten et al., 2020). Since 2018, analyzing SM data downloads is a promising new prospect to get access to adolescents' private and public interactions. According to the European General Data Protection Regulation (GDPR; https://gdpr-info.eu/), to which all large SM platforms comply, each platform is legally mandated to provide its European users with their SM archives upon request 
(Boeschoten et al., 2020). These so-called data download packages offer unprecedented opportunities to get insight in the content of private or semipublic social media interactions and their potential consequences for adolescents' psychosocial functioning (Griffioen et al., 2020), and, thus, they are an important avenue for future research.

\section{The Important Role of Parents and Educators}

Adolescents' positive SM experiences outweighed their negative SM experiences by a factor of three to one. More importantly, most adolescents (78\%) also experienced positive effects of these SM experiences on their self-esteem, whereas only $3 \%$ experienced negative effects of their SM experiences. Our results may be reassuring news for parents and educators. However, this positivity bias in SM experiences, along with the predominantly positive effects of these experiences, does not prevent the occasional occurrence of negative experiences, and their resulting negative impact on adolescents' self-esteem. Such negative experiences, and the subsequent drops in self-esteem, are functional. They help adolescents regulating subsequent self-esteem fluctuations, thereby contributing to the longer-term development of a stable self-esteem (MacDonald \& Leary, 2012). Therefore, our results do not give reason to keep adolescents away from SM, which they avidly use to interact with their friends and to experiment with their developing identity (Nesi et al., 2018). However, not only the negative susceptibles (i.e., adolescents who experienced mainly negative effects), but all adolescents need their parents and educators to help them prevent, or cope with negative SM experiences. Helping adolescents prevent or cope with negative feedback, social exclusion, or cyberbullying, and explaining them that the SM world is not as perfect as it often appears, should be essential components of today's parenting and school-based digital literacy programs.

\section{References}

Adachi, P., \& Willoughby, T. (2015). Interpreting effect sizes when controlling for stability effects in longitudinal autoregressive models: Implications for psychological science. European Journal of Developmental Psychology, 12(1), 116-128. https://doi.org/10.1080/17405629.2014 .963549

Asparouhov, T., Hamaker, E. L., \& Muthén, B. (2018). Dynamic structural equation models. Structural Equation Modeling, 25(3), 359-388. https:// doi.org/10.1080/10705511.2017.1406803

Barthorpe, A., Winstone, L., Mars, B., \& Moran, P. (2020). Is social media screen time really associated with poor adolescent mental health? A time use diary study. Journal of Affective Disorders, 274, 864-870. https:// doi.org/10.1016/j.jad.2020.05.106

Beyens, I., Pouwels, J. L., van Driel, I. I., Keijsers, L., \& Valkenburg, P. M. (2020). The effect of social media on well-being differs from adolescent to adolescent. Scientific Reports, 10(1), Article 10763. https://doi.org/10 .1038/s41598-020-67727-7

Blomfield Neira, C. J., \& Barber, B. L. (2014). Social networking site use: Linked to adolescents' social self-concept, self-esteem, and depressed mood. Australian Journal of Psychology, 66(1), 56-64. https://doi.org/10 .1111/ajpy.12034

Boeschoten, L., Ausloos, J., Moeller, J., Araujo, T., \& Oberski, D. L. (2020). Digital trace data collection through data donation. arXiv preprint arXiv:2011.09851.

Bronfenbrenner, U. (2005). The bioecological theory of human development. In U. Bronfenbrenner (Ed.), Making human beings human:
Bioecological perspectives on human development (pp. 6963-6970). Sage Publications.

Burke, M., Marlow, C., \& Lento, T. (2010, April 10-15). Social network activity and social well-being. Proceedings of the SIGCHI Conference on Human Factors in Computing Systems, Atlanta, GA, United States.

Cingel, D. P., \& Olsen, M. K. (2018). Getting over the hump: Examining curvilinear relationships between adolescent self-esteem and Facebook use. Journal of Broadcasting \& Electronic Media, 62(2), 215-231. https:// doi.org/10.1080/08838151.2018.1451860

Crocker, J., \& Brummelman, E. (2018). The self: Dynamics of persons and their situations. In K. Deaux \& M. Snyder (Eds.), The Oxford handbook of personality and social psychology (pp. 265-287). Oxford University Press. https://doi.org/10.1093/oxfordhb/9780190224837.013.11

Crocker, J., Luhtanen, R. K., Cooper, M. L., \& Bouvrette, A. (2003). Contingencies of self-worth in college students: Theory and measurement. Journal of Personality and Social Psychology, 85(5), 894-908. https:// doi.org/10.1037/0022-3514.85.5.894

Crocker, J., \& Wolfe, C. T. (2001). Contingencies of self-worth Psychological Review, 108(3), 593-623. https://doi.org/10.1037/0033-295X.108 .3 .593

Escobar-Viera, C. G., Shensa, A., Bowman, N. D., Sidani, J. E., Knight, J., James, A. E., \& Primack, B. A. (2018). Passive and active social media use and depressive symptoms among United States adults. Cyberpsychology, Behavior, and Social Networking, 21(7), 437-443. https://doi.org/10 $.1089 /$ cyber.2017.0668

Gorrese, A., \& Ruggieri, R. (2013). Peer attachment and self-esteem: A meta-analytic review. Personality and Individual Differences, 55(5), 559568. https://doi.org/10.1016/j.paid.2013.04.025

Griffioen, N., Rooij, M., Lichtwarck-Aschoff, A., \& Granic, I. (2020). Toward improved methods in social media research. Technology, Mind, and Behavior, 1(1). https://doi.org/10.1037/tmb0000005

Harter, S. (2012). The construction of the self: Developmental and sociocultural foundations. Guilford.

Harter, S., \& Whitesell, N. R. (2003). Beyond the debate: Why some adolescents report stable self-worth over time and situation, whereas others report changes in self-worth. Journal of Personality, 71(6), 1027-1058. https://doi.org/10.1111/1467-6494.7106006

Huang, C. (2017). Time spent on social network sites and psychological well-being: A meta-analysis. Cyberpsychology, Behavior, and Social Networking, 20(6), 346-354. https://doi.org/10.1089/cyber.2016.0758

Kelly, Y., Zilanawala, A., Booker, C., \& Sacker, A. (2019). Social media use and adolescent mental health: Findings from the UK Millennium Cohort Study. EClinicalMedicine, 6, 59-68. https://doi.org/10.1016/j.eclinm .2018.12.005

Kernis, M. H. (2003). Toward a conceptualization of optimal self-esteem. Psychological Inquiry, 14(1), 1-26. https://doi.org/10.1207/S1532796 5PLI1401_01

Kernis, M. H. (2005). Measuring self-esteem in context: The importance of stability of self-esteem in psychological functioning. Journal of Personality, 73(6), 1569-1605. https://doi.org/10.1111/j.1467-6494.2005.00359.x

Košir, K., Horvat, M., Aram, U., Jurinec, N., \& Tement, S. (2016). Does being on Facebook make me (feel) accepted in the classroom? The relationships between early adolescents' Facebook usage, classroom peer acceptance and self-concept. Computers in Human Behavior, 62, 375-384. https://doi.org/10.1016/j.chb.2016.04.013

Kraut, R., Kiesler, S., Boneva, B., Cummings, J., Helgeson, V., \& Crawford, A. (2002). Internet paradox revisited. Journal of Social Issues, 58(1), 4974. https://doi.org/10.1111/1540-4560.00248

Leary, M. R., \& Baumeister, R. F. (2000). The nature and function of selfesteem: Sociometer theory. Advances in Experimental Social Psychology, 32, 1-62. https://doi.org/10.1016/S0065-2601(00)80003-9

Lerner, R. M., \& Lerner, J. V. (2019). An idiographic approach to adolescent research: Theory, method, and application. In L. B. Hendry \& M. Kloep (Eds.), Reframing adolescent research (pp. 25-38). Routledge. 
Liu, D., \& Baumeister, R. F. (2016). Social networking online and personality of self-worth: A meta-analysis. Journal of Research in Personality, 64, 79-89. https://doi.org/10.1016/j.jrp.2016.06.024

MacDonald, G., \& Leary, M. R. (2012). Individual differences in selfesteem. In M. R. Leary \& J. P. Tangney (Eds.), Handbook of self and identity (2nd ed., pp. 354-377). Guilford Press.

Maciejewski, D. F., Keijsers, L., van Lier, P. A. C., Branje, S. J. T., Meeus, W. H. J., \& Koot, H. M. (2019). Most fare well-But some do not: Distinct profiles of mood variability development and their association with adjustment during adolescence. Developmental Psychology, 55(2), 434-448. https://doi.org/10.1037/dev0000650

McNeish, D., \& Hamaker, E. L. (2020). A primer on two-level dynamic structural equation models for intensive longitudinal data in Mplus. Psychological Methods, 25(5), 610-635. https://doi.org/10.1037/me t0000250

Meeus, A., Beullens, K., \& Eggermont, S. (2019). Like me (please?): Connecting online self-presentation to pre- and early adolescents' selfesteem. New Media \& Society, 21(11-12), 2386-2403. https://doi.org/10 $.1177 / 1461444819847447$

Meier, L. L., Orth, U., Denissen, J. J. A., \& Kühnel, A. (2011). Age differences in instability, contingency, and level of self-esteem across the life span. Journal of Research in Personality, 45(6), 604-612. https:// doi.org/10.1016/j.jrp.2011.08.008

Nesi, J., Choukas-Bradley, S., \& Prinstein, M. J. (2018). Transformation of adolescent peer relations in the social media context: Part 1-A theoretical framework and application to dyadic peer relationships. Clinical Child and Family Psychology Review, 21(3), 267-294. https://doi.org/10.1007/ s10567-018-0261-x

Nesselroade, J. R. (1991). The warp and the woof of the developmental fabric. In R. M. Downs, L. S. Liben, \& D. Palermo (Eds.), Visions of aesthetics, the environment \& development: The legacy of Joachim $F$. Wohlwill (pp. 213-240). Lawrence Erlbaum.

Orben, A. C., \& Dunbar, R. I. M. (2017). Social media and relationship development: The effect of valence and intimacy of posts. Computers in Human Behavior, 73, 489-498. https://doi.org/10.1016/j.chb.2017.04.006

Orth, U., \& Robins, R. W. (2014). The development of self-esteem. Current Directions in Psychological Science, 23(5), 381-387. https://doi.org/10 $.1177 / 0963721414547414$

Pew Research Center. (2018). Teens social media \& technology 2018. https://www.pewresearch.org/internet/2018/05/31/teens-social-media-te chnology-2018/

Primack, B. A., Karim, S. A., Shensa, A., Bowman, N., Knight, J., \& Sidani, J. E. (2019). Positive and negative experiences on social media and perceived social isolation. American Journal of Health Promotion, 33(6), 859-868. https://doi.org/10.1177/0890117118824196

Proctor, C., Linley, P., \& Maltby, J. (2009). Youth life satisfaction: A review of the literature. Journal of Happiness Studies, 10(5), 583-630. https:// doi.org/10.1007/s10902-008-9110-9

Reeves, B., Ram, N., Robinson, T. N., Cummings, J. J., Giles, C. L., Pan, J., Chiatti, A., Cho, M., Roehrick, K., Yang, X., Gagneja, A., Brinberg, M., Muise, D., Lu, Y., Luo, M., Fitzgerald, A., \& Yeykelis, L. (2021). Screenomics: A framework to capture and analyze personal life experiences and the ways that technology shapes them. Human-Computer Interaction, 36(2), 150-201. https://doi.org/10.1080/07370024.2019.1578652

Reinecke, L., \& Trepte, S. (2014). Authenticity and well-being on social network sites: A two-wave longitudinal study on the effects of online authenticity and the positivity bias in SNS communication. Computers in Human Behavior, 30, 95-102. https://doi.org/10.1016/j.chb.2013.07.030

Robins, R. W., Hendin, H. M., \& Trzesniewski, K. H. (2001). Measuring global self-esteem: Construct validation of a single-item measure and the Rosenberg self-esteem scale. Personality and Social Psychology Bulletin, 27(2), 151-161. https://doi.org/10.1177/0146167201272002
Rodgers, R. F., Slater, A., Gordon, C. S., McLean, S. A., Jarman, H. K., \& Paxton, S. J. (2020). A biopsychosocial model of social media use and body image concerns, disordered eating, and muscle-building behaviors among adolescent girls and boys. Journal of Youth and Adolescence, 49(2), 399-409. https://doi.org/10.1007/s10964-019-01190-0

Rosenberg, M. (1986). Self-concept and psychological well-being in adolescence. In R. L. Leahy (Ed.), The development of the self (pp. 205-246). Academic Press.

Steinsbekk, S., Wichstrøm, L., Stenseng, F., Nesi, J., Hygen, B. W., \& Skalická, V. (2021). The impact of social media use on appearance selfesteem from childhood to adolescence: A 3-wave community study. Computers in Human Behavior, 114, Article 106528. https://doi.org/10 .1016/j.chb.2020.106528

Valkenburg, P. M., Beyens, I., Pouwels, J. L., van Driel, I. I., \& Keijsers, L. (2021a). Social media and adolescents' self-esteem: Heading for a personspecific media effects paradigm. Journal of Communication, 71(1), 56-78. https://doi.org/10.1093/joc/jqaa039

Valkenburg, P. M., Beyens, I., Pouwels, J. L., van Driel, I. I., \& Keijsers, L. (2021b). Social media browsing and adolescent well-being: Challenging the "Passive Social Media Use Hypothesis." PsyArXiv. https://doi.org/10 .31234/osf.io/gzu3y

Valkenburg, P. M., Koutamanis, M., \& Vossen, H. G. M. (2017). The concurrent and longitudinal relationships between adolescents' use of social network sites and their social self-esteem. Computers in Human Behavior, 76, 35-41. https://doi.org/10.1016/j.chb.2017.07.008

Valkenburg, P. M., \& Peter, J. (2011). Online communication among adolescents: An integrated model of its attraction, opportunities, and risks. The Journal of Adolescent Health, 48(2), 121-127. https://doi.org/10 .1016/j.jadohealth.2010.08.020

Valkenburg, P. M., \& Peter, J. (2013). The differential susceptibility to media effects model. Journal of Communication, 63(2), 221-243. https://doi.org/ 10.1111/jcom. 12024

Valkenburg, P. M., Peter, J., \& Walther, J. B. (2016). Media effects: Theory and research. Annual Review of Psychology, 67(1), 315-338. https:// doi.org/10.1146/annurev-psych-122414-033608

Valkenburg, P. M., Pouwels, J. L., Beyens, I., van Driel, I. I., \& Keijsers, L. (2021). Dataset belonging to Valkenburg et al (2021). Adolescents' social media experiences and their self-esteem: A person-specific susceptibility perspective. University of Amsterdam/Amsterdam University of Applied Sciences. https://doi.org/10.21942/uva.14095971

van Driel, I. I., Pouwels, J. L., Beyens, I., Keijsers, L., \& Valkenburg, P. M. (2019). Posting, scrolling, chatting, and snapping: Youth (14-15) and social media in 2019. Center for Research on Children, Adolescents, and the Media.

van Roekel, E., Keijsers, L., \& Chung, J. M. (2019). A review of current ambulatory assessment studies in adolescent samples and practical recommendations. Journal of Research on Adolescence, 29(3), 560-577. https://doi.org/10.1111/jora.12471

Vonk, R., \& Smit, H. (2012). Optimal self-esteem is contingent: Intrinsic versus extrinsic and upward versus downward contingencies. European Journal of Personality, 26(3), 182-193. https://doi.org/10.1002/ per.817

Waterloo, S. F., Baumgartner, S. E., Peter, J., \& Valkenburg, P. M. (2018). Norms of online expressions of emotion: Comparing Facebook, Twitter, Instagram, and WhatsApp. New Media \& Society, 20(5), 1813-1831. https://doi.org/10.1177/1461444817707349

Received February 24, 2021

Revision received May 3, 2021

Accepted May 5, 2021 